\title{
GENERALIZED MORSE SEQUENCES ON $n$ SYMBOLS
}

\author{
JOHN C. MARTIN 1
}

\begin{abstract}
A class of bisequences on $\mathrm{n}$ symbols is constructed which includes the generalized Morse sequences introduced by Keane. The topological structure and endomorphisms of the resulting minimal symbolic flows are described.
\end{abstract}

Introduction. We construct a class of bisequences on $s$ symbols $(s \geq 2)$ which contains the generalized Morse sequences on two symbols described by Keane in [9]. The orbit-closures of these sequences in the shift dynamical system on $s$ symbols are point-distal symbolic flows, and we consider their topological structure. In our main theorems, we describe the maximal equicontinuous factor of such a flow; we prove that the symbolic flow is an isometric extension of an almost automorphic extension of its maximal equicontinuous factor; and we determine all endomorphisms of the flow. These theorems generalize results of Coven, Keane, and the author on substitution minimal sets. For basic definitions, the reader is referred to [3], [5], and [11]. The duthor would like to thank the referee for a helpful suggestion regarding the proof of Theorem 7.

1. Construction. Let $s$ be an integer greater than 1 , and let $S=\{0,1, \ldots, s$ - 1\}. $B_{k}$ will denote the set of $k$-blocks over $S, X$ the set of sequences over $S$ (i.e., functions from the nonnegative integers to $S$ ), and $\Omega$ the set of bisequences over $S$. If $A \in B_{k}, C \in B_{m}, A C \in B_{k+m}$ is defined by $A C$ $=A(0) \cdots A(k-1) C(0) \cdots C(m-1)$. For $x$ an element of $B_{k}, X$, or $\Omega$, $x(j, m)$ will denote the $m$-block $x(j) x(j+1) \cdots x(j+m-1)$. If $A \in B_{k}$, let $L(A)=k$.

Now we take $G=\left\{\sigma_{0}, \sigma_{1}, \ldots, \sigma_{s-1}\right\}$ to be any subgroup of the group of permutations of $\{0,1, \ldots, s-1\}$, where $\sigma_{0}$ is the identity. Thus $\sigma_{i}$ may be considered as a function from $B_{k}, X$, or $\Omega$ to itself. If $A \in B_{j}, C \in B_{k}$, define

$$
A \times C=\sigma_{C(0)} A \sigma_{C(1)} A \cdots \sigma_{C(k-1)} A \in B_{j k} .
$$

For each $j \geq 0$, let $m_{j} \geq 2$, and let $b_{j}$ be an element of $B_{m_{j}}$ with $b_{j}(0)=0$. Then we may define an element of $X$ as follows:

Presented to the Society, January 23, 1975; received by the editors March 16, 1975 and, in revised form, May 1, 1975.

AMS (MOS) subject classifications (1970). Primary 54H20; Secondary 28A65.

Key words and phrases. Almost periodic, symbolic flow, distal point, equicontinuous factor, almost automorphic extension, isometric extension, substitution minimal set.

${ }^{1}$ Research supported in part by NSF Institutional Grant for Science, GU 6315, North Dakota State University. 


$$
x=\cdots\left(\left(b_{0} \times b_{1}\right) \times b_{2}\right) \times \cdots .
$$

For $t \geq 0$, let $c_{t}=\left(\cdots\left(\left(b_{0} \times b_{1}\right) \times b_{2}\right) \times \cdots b_{t}\right)$; let $n_{t}=L\left(c_{t}\right)=m_{0} \cdots m_{t}$. We observe that for each $t$,

$$
x=c_{t} \sigma_{i_{1}}\left(c_{t}\right) \sigma_{i_{2}}\left(c_{t}\right) \cdots
$$

for some sequence $i_{1}, i_{2}, \ldots$ satisfying $0 i_{1} i_{2} \cdots i_{m_{t+1}-1}=b_{t+1}$.

We assume that both $b_{0}$ and, for each $t$, the associated sequence $i_{1} i_{2} \cdots$ in (1) contain every symbol in $S$.

LEMMA 1. $x$ is a periodic sequence if and only if, for some $t$, the sequence $i_{1} i_{2} \cdots$ in (1) is periodic of period $s$.

For $s=2$, this is Lemma 1 of [9]. The proof in the general case is a straightforward, if somewhat tedious, adaptation of that in [9].

We let $T: \Omega \rightarrow \Omega$ denote the shift transformation: $(T \omega)(n)=\omega(n+1)(n$ $\in Z)$.

Proposition 2. There is an almost periodic point $\omega$ in the shift dynamical system $(\Omega, T)$ with $\omega(n)=x(n)(n \geq 0)$.

Proof. Let $k \geq 0$, and choose $t$ so that $n_{t} \geq k$; by (2) we may find $u$ so that each $n_{t}$-block $\sigma_{i}\left(c_{t}\right)$ appears in $c_{u}$. Now by (1), $x=c_{u} \sigma_{i_{1}}\left(c_{u}\right) \cdots$. Thus any $2 n_{u}$-block of $x$ contains $\sigma_{j}\left(c_{u}\right)$ for some $j$; but $c_{u}$ contains the $n_{t}$-block $\sigma_{j}^{-1}\left(c_{t}\right)$. Thus every $2 n_{u}$-block of $x$ contains $x(0, k)$. It is now an easy matter to extend $x$ to an almost periodic bisequence $\omega$. Q.E.D.

We assume from now on that $\omega$ is a fixed, nonperiodic, almost periodic bisequence which extends $x$. We call $\omega$ a generalized Morse sequence (though in [9] the term is reserved for sequences of this type which are strictly transitive). We denote by $X_{\omega}$ the orbit-closure of $\omega$ under $T$.

2. A basic lemma on the block structure of $x$. For $t \geq 0$, and $A$ a $k$-block of $x, A$ is said to be determined to order $t$ if, whenever $A=x(n, k)=x(m, k)$, then $m=n\left(\bmod n_{t}\right)$.

Lemma 3. For any $t$, there is a $k$ so that every $k$-block of $x$ is determined to order $t$.

Proof. It is sufficient to find some $t$ for which the statement holds. Choose $t$ large enough so that $c_{t}=A B C$, where $A$ and $C$ both contain every symbol in $S$, and $\max (L(A), L(C)) \leq n_{t} / s$. Now, by redefining $m_{0}$, we may as well assume $t=0$.

We show that some $c_{u}$ is determined to order 0 . If not, then for each $u$, $c_{u}=x\left(a_{u}, n_{u}\right)$ for some $a_{u} \neq 0\left(\bmod n_{0}\right)$. By considering a subsequence, we may assume that for some $a\left(0<a<n_{0}\right), a_{u}=n_{0}-a\left(\bmod n_{0}\right)$ for each $u$. This implies that $x=D \sigma_{j_{1}}\left(c_{0}\right) \sigma_{j_{2}}\left(c_{0}\right) \cdots$, where $D \in B_{a}$, and any initial portion of $\sigma_{j_{1}}\left(c_{0}\right) \sigma_{j_{2}}\left(c_{0}\right) \cdots$ appears in $x$ beginning at some position equal to $0 \bmod n_{0}$. We consider two cases.

(i) $n_{0} / s \leq a \leq(1-1 / s) n_{0}$. We have $x=c_{0} \sigma_{i_{1}}\left(c_{0}\right) \sigma_{i_{2}}\left(c_{0}\right) \cdots$. Consider the sequence $j_{1} i_{1} j_{2} i_{2} \cdots$, and take any $j_{k}$. The last $a$-block of $\sigma_{j_{k}}\left(c_{0}\right)$ is the first $a$ - 
block of $\sigma_{i_{k}}\left(c_{0}\right)$, and it contains $\sigma_{j_{k}}(C)$ since $L(C) \leq n_{0} / s$. Thus it contains every symbol in $S$. This implies that $i_{k}$ is determined uniquely by $j_{k}$. Similarly, given $i_{k}, j_{k}$ is determined. Thus the sequence $j_{1} i_{1} \cdots$ is periodic, since every symbol has a unique successor; but this contradicts the fact that $x$ is not periodic.

(ii) $a<n_{0} / s$ or $a>(1-1 / s) n_{0}$. Again $c_{0} \sigma_{i_{1}}\left(c_{0}\right) \cdots=D \sigma_{j_{1}}\left(c_{0}\right) \cdots$, where $D \in B_{a}$. This implies that for $k, m \geq 0, x\left(k n_{0}+m a, n_{0}\right)$ is a block of the form $\sigma_{i}\left(c_{0}\right)$. It follows that if $d$ is the greatest common divisor of $n_{0}$ and $a$, then for $k \geq 0, x\left(k d, n_{0}\right)$ is of the form $\sigma_{i}\left(c_{0}\right)$. Now let the distinct $d$-blocks of the form $x(k d, d)$ be denoted $A_{1}, A_{2}, \ldots, A_{r}$. Then $x=A_{i_{1}} A_{i_{2}} \cdots$, and for each $k$ the nonperiodic sequence $i_{1} i_{2} \cdots$ contains at least $k+1$ distinct $k$-blocks. Letting $k=n_{0} / d$, we obtain $k>s$, using our assumption on $a$. We have shown that there are more than $s$ distinct $n_{0}$-blocks of the form $x\left(k d, n_{0}\right)$; but every such block is $\sigma_{i}\left(c_{0}\right)$ for some $i$. This contradiction completes the proof. Q.E.D.

For $s=2$, the above lemma is approximately Lemma 5 of [9].

We list some simple consequences of Lemma 3.

LEMMA 4. (a) For each $t, \omega\left(-n_{t}, n_{t}\right)=\sigma_{i}\left(c_{t}\right)$ for some $i$.

(b) If $y \in X$, and $T^{j_{k}} y$ converges, then for each $t, j_{k}-j_{m}=0\left(\bmod n_{t}\right)$ for all sufficiently large $k$ and $m$.

3. Equicontinuous factors of $\left(X_{\omega}, T\right)$. We denote by $(Z(k), 1)$ the minimal rotation $z \rightarrow z+1$ on the cyclic group $Z(k)$ of order $k$. If $\mathbf{a}=\left(a_{0}, a_{1}, \ldots\right)$, where $a_{i} \geq 2$, and $d_{i}=a_{0} a_{1} \cdots a_{i}$, we let $(\Delta(\mathbf{a}), 1)$ be the minimal equicontinuous flow $z \rightarrow z+1$ on the group $\Delta(\mathbf{a})$ of a-adic integers-that is the inverse limit of the groups $Z\left(d_{i}\right)$. (Here "1" means the element $(1,1, \ldots) \in \Delta(\mathbf{a})$.)

Proposition 5. There is a flow homomorphism $f$ from $\left(X_{\omega}, T\right)$ to $(\Delta(\mathbf{m}), 1)$, where $\mathbf{m}=\left(m_{0}, m_{1}, \ldots\right)$, such that if $z=\left(z_{0}, z_{1}, \ldots\right) \in \Delta(\mathbf{m}), f(y)=z$ if and only if $y\left(-z_{t}, n_{t}\right)$ is of the form $\sigma_{i}\left(c_{t}\right)$ for every $t$. For $i \in S, y \in X, f\left(\sigma_{i} y\right)$ $=f(y)$. If $z$ is not in the orbit of $O$ in $\Delta(\mathbf{m})$ and $y \in f^{-1}(z), f^{-1}(z)$ $=\left\{\sigma_{i} y: i \in S\right\}$.

Proof. It is clear from (b) of Lemma 4 that for each $t$, the function $f_{t}$ defined on the orbit of $\omega$ by $f_{t}\left(T^{k} \omega\right)=k \in Z\left(n_{t}\right)$ extends continuously to a flow homomorphism $f_{t}:\left(X_{\omega}, T\right) \rightarrow\left(Z\left(n_{t}\right), 1\right)$, and the maps $f_{t}$ induce a homomorphism $f$. Suppose $f(y)=z$, and let $T^{j_{k}} \omega \rightarrow y$. For each $t, j_{k}-j_{m}$ $=0\left(\bmod n_{t}\right)$ for $k, m \geq k_{0}$. Thus for $k \geq k_{0}, T^{j_{k}} \omega$ and $y$ have blocks of the form $\sigma_{i}\left(c_{t}\right)$ in the same positions, and $f_{t}\left(T^{j_{k}} \omega\right)=z_{t}$. But clearly $T^{j_{k}} \omega\left(-z_{t}, n_{t}\right)$ is a block of the desired form. The remainder of the first statement is proved similarly. The second statement is now obvious. Finally, if $f(y)=f(w)=z$ $=\left(z_{0}, z_{1}, \ldots\right) \in \Delta(\mathbf{m})$, then for each $t, y\left(-z_{t}, n_{t}\right)=\sigma_{i_{t}}\left(w\left(-z_{t}, n_{t}\right)\right)$. But since $c_{0}$ contains every symbol, all the $i_{t}$,s are equal, say to $i$. If $z$ is not in the orbit of 0 , it follows that $y=\sigma_{i} w$. Q.E.D.

Corollary 6. If $z \in \Delta(\mathbf{m})$ is not an integer, any point in $f^{-1}(z)$ is a distal point. Hence $\left(X_{\omega}, T\right)$ is point-distal.

If $(X, T)$ and $(Z, T)$ are discrete flows, then $(X, T)$ is called a (proper) AI extension of $(Z, T)$ if $(X, T)$ is a (nontrivial) isometric extension of some $(Y, T)$, which is an almost automorphic extension of $(Z, T)$. We denote by $\left(X_{\omega}^{*}, T\right)$ the maximal equicontinuous factor of $\left(X_{\omega}, T\right)$. 
THeOREM 7. $\left(X_{\omega}^{*}, T\right)$ is isomorphic to $\left(\Delta\left(\mathbf{m}^{\prime}\right), 1\right)$, for some $\mathbf{m}^{\prime}=\left(m_{0}{ }^{\prime}, m_{1}\right.$, $\left.m_{2}, \ldots\right)$, where $m_{0}^{\prime}=m_{0} r$ for some divisor $r$ of $s .\left(X_{\omega}, T\right)$ is a proper AI extension of $\left(X_{\omega}^{*}, T\right)$.

Proof. We first show that $\left(X_{\omega}, T\right)$ is an AI extension of $(\Delta(\mathbf{m}), 1)$. Let $(Y, T)=\left(X_{\omega}, T\right) / G$ (using Proposition 5). We then have $\left(X_{\omega}, T\right) \stackrel{g}{\rightarrow}(Y, T)$ $\stackrel{h}{\rightarrow}(\Delta(\mathbf{m}), 1)$, where $h g=f$, and $h^{-1}(z)$ is a single point if $z$ is not in the orbit of 0 . If we define $R$ on $\left\{\left(x_{1}, x_{2}\right): g\left(x_{1}\right)=g\left(x_{2}\right)\right\}$ by $R(x, y)=0$ if $x=y$, $R(x, y)=1$ if $x \neq y$, then $R$ is continuous. Thus $\left(X_{\omega}, T\right)$ is an isometric extension of $(Y, T)$.

Now from [10, Theorem 8.11], $\left(X_{\omega}, T\right)$ is an AI extension of $\left(X_{\omega}^{*}, T\right)$. We obtain the diagram

$$
\left(X_{\omega}, T\right) \stackrel{\rho}{\longrightarrow}(W, T) \stackrel{\tau}{\longrightarrow}\left(X_{\omega}^{*}, T\right) \stackrel{\pi}{\longrightarrow}(\Delta(\mathbf{m}), 1),
$$

where $\pi \tau \rho=f, \pi$ is $r$-to-one for some divisor $r$ of $s$, and $\rho$ is $s / r$-to-one. Thus, using the criterion for two groups $\Delta(\mathbf{m})$ and $\Delta\left(\mathbf{m}^{\prime}\right)$ to be isomorphic, we see that $X_{\omega}^{*}$ is isomorphic to $\Delta\left(\mathbf{m}^{\prime}\right)$, where $\mathbf{m}^{\prime}$ is of the desired form. Finally, it can be seen that $r \neq s$, so that $\rho$ is not 1-1. Q.E.D.

COROLlaRy 8. If either (a) $s$ is prime; or (b) every prime factor of $s$ appears in infinitely many $m_{i}$ 's, then $\left(X_{\omega}^{*}, T\right)$ is isomorphic to $(\Delta(\mathbf{m}), 1)$.

It is possible, however, to construct for each nonprime $s$ examples where $\left(X_{\omega}^{*}, T\right)$ is not isomorphic to $(\Delta(\mathbf{m}), 1)$.

COROLlary 9. If $Z$ is any infinite, compact, zero-dimensional, monothetic group, 1 is a generator for $Z$, and $s \geq 2$, there is a generalized Morse sequence $\omega$ on s symbols with $\left(X_{\omega}^{*}, T\right) \simeq(Z, 1)$.

Proof. This is a simple consequence of the fact that any such $Z$ is isomorphic to $\Delta(\mathbf{m})$ for some $\mathbf{m}[7]$. Q.E.D.

\section{Endomorphisms of $\left(X_{\omega}, T\right)$.}

THEOREM 10. If $\psi$ is an endomorphism of $\left(X_{\omega}, T\right)$, then $\psi=T^{k} \sigma_{m}$ for some $k \in Z, m \in S$.

Proof. We let $B_{k}(\omega)$ denote the set of $k$-blocks of $\omega$. It is well known that for some integer $p$ and some $g: B_{j}(\omega) \rightarrow S$, the map $\phi=T^{p} \psi$ is the block map $g_{\infty}$ (see [6]). For $n \geq 1$, let $g_{n}: B_{j+n-1}(\omega) \rightarrow B_{n}(\omega)$ be the function induced by $g$, and choose $n \geq 2 n_{0}$ with $j+n-1=n_{t}$. Now for some unique $r(0 \leq r$ $\left.<n_{t}\right)$, each block $\phi(\omega)\left(r+i n_{t}, n_{t}\right)$ is of the form $\sigma_{j_{i}}\left(c_{t}\right)$. We assume $r \geq 2 n_{0}$; the other case is proved similarly. Then for each $i, \phi(\omega)\left(r+(i-1) n_{t}, n_{t}\right)$ is determined by $\omega\left(i n_{t}, n_{t}\right)$. Let $\phi_{1}=T^{r-n_{t}} \phi$. Then given either of the blocks $\phi_{1}(\omega)\left(i n_{t}, n_{t}\right)$ and $\omega\left(i n_{t}, n_{t}\right)$, the other is determined. Then for $u \geq t, \phi_{1}(\omega)$ - $\left(i n_{u}, n_{u}\right)$ is of the form $\sigma_{k_{i}}\left(c_{u}\right)$. (Otherwise, for some $a$ with $0<a<n_{u}$ and $a=0\left(\bmod n_{t}\right)$, we have $\phi_{1}(\omega)\left(a+i n_{u}, n_{u}\right)=\sigma_{k_{i}}\left(c_{u}\right)$ for each $i$, from which it follows that if $\omega\left(i n_{u}, n_{u}\right)=\sigma_{j_{i}}\left(n_{u}\right)$, the sequence $j_{0} k_{0} j_{1} k_{1} \cdots$ is periodic.) Hence, for some $m, \phi_{1}(\omega)\left(0, n_{u}\right)=\sigma_{m}\left(0, n_{u}\right)$ for each $u$, and thus $\phi_{1}=\sigma_{m}$. Q.E.D.

5. Remarks. We comment briefly on the almost automorphic flows $(Y, T)$ obtained in Theorem 7, in the special case when $G$ is the group generated by 
the cyclic permutation $\sigma_{1}: j \rightarrow j+1(\bmod s) .(Y, T)$ is isomorphic to a symbolic flow, and the map $g$ may be defined by $(g x)(n)=x(n)+x(n+1)$ $+\cdots+x(n+s-1)(\bmod s)$. This is similar to the construction discussed in [2] and [5]. $(Y, T)$ is always a strictly ergodic flow, and thus using the results of [9], we obtain examples of a two-to-one group extension of a strictly ergodic flow which is not strictly ergodic. It is also possible to show that the only endomorphisms of $(Y, T)$ are powers of the shift.

Certain special cases of this construction have been discussed extensively. By taking $b_{0}=b_{1}=\cdots$, and $G$ the cyclic group above, we obtain a substitution minimal set. If $s=2$, every continuous substitution minimal set can be obtained this way, and hence our main theorems generalize results of Coven and Keane in [1] and [2]. In another special case $(s=4)$, it is possible to obtain the strictly transitive sequence constructed by Kakutani in [8].

\section{REFERENCES}

1. E. M. Coven, Endomorphisms of substitution minimal sets, Z. Wahrscheinlichkeitstheorie und Verw. Gebiete 20 (1971/72), 129-133. MR 46 \#6332.

2. E. M. Coven and M. S. Keane, The structure of substitution minimal sets, Trans. Amer. Math. Soc. 162 (1971), 89-102. MR 44 \#2219.

3. R. Ellis, Lectures on topological dynamics, Benjamin, New York, 1969. MR 42 \#2463.

4. W. H. Gottschalk, Substitution minimal sets, Trans. Amer. Math. Soc. 109 (1963), 467-491. MR 32 \#8325.

5. W. H. Gottschalk and G. A. Hedlund, Topological dynamics, Amer. Math. Soc. Colloq. Publ., vol. 36, Amer. Math. Soc., Providence, R.I., 1955. MR 17, 650.

6. G. A. Hedlund, Endomorphisms and automorphisms of the shift dynamical system, Math. Systems Theory 3 (1969), 320-375. MR 41 \#510.

7. E. Hewitt and K. A. Ross, Abstract harmonic analysis. Vol. I: Structure of topological groups. Integration theory, group representations, Die Grundlehren der math. Wissenschaften, Band 115, Academic Press, New York; Springer-Verlag, Berlin, 1963. MR 28 \# 158.

8. S. Kakutani, Ergodic theory of shift transformations, Proc. Fifth Berkeley Sympos. Math. Statist. and Probability (Berkeley, Calif., 1965/66), vol. II: Contributions to Probability Theory, part 2, Univ. of California Press, Berkeley, Calif., 1967, pp. 405-414. MR 37 \#2943.

9. M. S. Keane, Generalized Morse sequences, Z. Wahrscheinlichkeitstheorie und Verw. Gebiete 10 (1968), 335-353. MR 39 \# 406.

10. J. C. Martin, Substitution minimal flows, Amer. J. Math. 93 (1971), 503-526. MR 45 \#9307.

11. W. A. Veech, Point-distal flows, Amer. J. Math. 92 (1970), 205-242. MR 42 \#2462.

Department of Mathematics, North Dakota State University, Fargo, North Dakota 58102 